\title{
Energy saving and solar electricity in fan-ventilated greenhouses
}

\author{
P. A. Davies ${ }^{\dagger}$, A. K. Hossain and G. Lychnos \\ School of Engineering and Applied Science \\ Aston University \\ Birmingham B4 7ET, UK \\ †corresponding author: p.a.davies@aston.ac.uk
}

\author{
C. Paton \\ Seawater Greenhouse Ltd \\ 2A Greenwood Road \\ London, E8 1AB, UK
}

Keywords: Fan and pad, solar energy, energy efficiency

\begin{abstract}
Greenhouse cultivation is an energy intensive process therefore it is worthwhile to introduce energy saving measures and alternative energy sources. Here we show that there is scope for energy saving in fan ventilated greenhouses. Measurements of electricity usage as a function of fan speed have been performed for two models of $1.25 \mathrm{~m}$ diameter greenhouse fans and compared to theoretical values. Reducing the speed can cut the energy usage per volume of air moved by more than $70 \%$. To minimize the capital cost of low-speed operation, a cooled greenhouse has been built in which the fan speed responds to sunlight such that full speed is reached only around noon. The energy saving is about $40 \%$ compared to constant speed operation. Direct operation of fans from solar-photovoltaic modules is also viable as shown from experiments with a fan driven by a brushless DC motor. On comparing the Net Present Value costs of the different systems over a 10 year amortization period (with and without a carbon tax to represent environmental costs) we find that sunlight-controlled system saves money under all assumptions about taxation and discount rates. The solar-powered system, however, is only profitable for very low discount rates, due to the high initial capital costs. Nonetheless this system could be of interest for its reliability in developing countries where mains electricity is intermittent. We recommend that greenhouse fan manufacturers improve the availability of energy-saving designs such as those described here.
\end{abstract}

\section{INTRODUCTION}

No issue is more relevant today than the sustainability of processes designed or managed by humans. Among such processes, those concerned with the supply of food and clean water are among the most critical. Cultivation in greenhouses has much potential here, as it encourages the efficient use of land and water and can provide food all year round. However, greenhouses are an energy-intensive form of cultivation especially when artificial heating, cooling or lighting is used (Campiglia et al., 2007). Therefore energy conservation in greenhouses and the use of energy from non-fossil sources is of great interest. Recent reviews of heating and cooling technologies for greenhouses, based on solar and other forms of energy, have been provided by Sethi and Sharma (2008 and 2007).

The focus here is on energy saving through the improved design of fan ventilation systems. Our objective is to explore and quantify, through mathematical models and practical experiments, the savings achievable. We will than evaluate the proposed designs from economic and environmental perspectives. 


\section{THEORETICAL POTENTIAL FOR ENERGY SAVING}

That there is scope of saving energy in fan ventilated systems can be appreciated by taking a simple example of a standard fan-and-pad type greenhouse measuring $9 \mathrm{~m}$ wide by $30 \mathrm{~m}$ long and having a volume of $1080 \mathrm{~m}^{3}$. Following the guidelines cited in Hanan (1998), this greenhouse should be ventilated with an air flow of about $10.8 \mathrm{~m}^{3} \mathrm{~s}^{-1}$, corresponding to 0.6 air changes per minute. To achieve such a flow, let us use a $1.27 \mathrm{~m}$ diameter (50") box fan, drawing about $750 \mathrm{~W}$ of electrical power (1hp). The air enters the greenhouse through cooling pads $1.8 \mathrm{~m}$ high and $0.1 \mathrm{~m}$ thick, occupying the end wall, giving a pad area of $16.2 \mathrm{~m}^{2}$ and an air velocity of $0.67 \mathrm{~ms}^{-1}$ at the pad face. Correspondingly, the pressure drop for a modern pad is below $10 \mathrm{~Pa}$ (Munters, 2005). Based on the first law of thermodynamics (Rogers and Mayhew, 1967), the theoretical minimum rate of work that is needed to move the air through the pad is obtained simply by multiplying the pressure drop by the volumetric flow rate, giving just $108 \mathrm{~W}$.

This result is much smaller than the electrical power of $750 \mathrm{~W}$ used by the fan, suggesting that most of this power is wasted in the sense that it is dissipated in processes other than drawing the air through the pad. However, using conventional equipment, the only readily available option to reduce the power input is to replace the box fan with a cone fan. This would cut the electricity consumption by about $20 \%$ - a worthwhile improvement but one which still falls a long way short of what is theoretically possible.

What then is the cause of the inefficiency in this fan-and-pad system? To gain some insight into this we refer to the schematic of Fig. 1 which shows a fan drawing air into a greenhouse through an intake which could be an evaporative pad as above. Alternatively the intake could be a ventilation louver, insect exclusion net or duct.

Fig. 1 can be analysed with the help of Bernouilli's equation applied to the fan orifice, resulting in the following equation for the minimum power input $\dot{W}_{\min }$ required by the fan.

$$
\dot{W}_{\text {min }}=0.5 \rho \dot{V}^{3} c_{l} / A_{\text {intake }}^{2}+0.5 \rho \dot{V}^{3} / A_{\text {fan }}^{2}
$$

where $\rho$ is the density of the air (assumed to be a constant due to the small pressure and temperature variations through the system), $c_{l}$ is a pressure loss coefficient for the intake component, $\dot{V}$ is the volumetric air flow rate and $A_{\text {intake }}$ and $A_{f a n}$ are the areas transverse to the direction of flow. In arriving at Eq. 1 we have assumed the air velocity inside the greenhouse growing area to be almost zero, as in practical arrangements it is indeed very much smaller than at the fan.

The first term on the right of this equation corresponds to the pressure drop across the intake component and the second to the pressure drop across the fan orifice. Applied to the example given at the beginning of this section, these terms take values of $108 \mathrm{~W}$ and $430 \mathrm{~W}$ respectively. The large value of the second term illustrates the paradox that arises in ventilation. The fan is there to drive air flow through the system - but at the same time it becomes the main restriction to flow by virtue of its small orifice. (Note that more precise modelling of the air flow at the fan and outlet is possible, but this would not alter this general conclusion).

This paradox can be overcome by increasing the area of the fan, using more fans or adding a diffusion cone. (A diffusion cone works by recovering energy from the exiting air jet; correspondingly $A_{f a n}$ in Eq. 1 is replaced by the area measured at the outlet 
of the cone). To see how much energy can be saved by such measures, we must first note that Eq. 1 corresponds to an ideal situation because it does not take into account some of the losses that will occur in a real fan, due to the fact that not all the work at the shaft of the fan is converted into useful air movement; further losses will occur in the electric motor and reduction belt.

Nonetheless, an equation similar to Eq. 1 applies in practice. This is generally known as the 'fan law' which states that the power needed to drive the fan will be proportional to the cube of the fan speed $\omega$ measured in radians per second (Bleier, 1997).

$$
\dot{W}=k_{1} \omega^{3}
$$

where $k_{1}$ is a constant of proportionality. Meanwhile, the flow rate is observed to be proportional to $\omega$.

$$
\dot{V}=k_{2} \omega
$$

where $k_{2}$ is another constant. It follows from Eqs. 2 and 3 that the specific energy $e$ needed to move one cubic metre of air through the system increases with the square of the speed:

$$
e=\dot{W} / \dot{V}=\left(k_{1} / k_{2}\right) \omega^{2}
$$

Halving the speed will therefore reduce the power input eight-fold, halve the air flow and reduce the specific energy by a factor of four. The penalty to pay in achieving this energy saving is that fans of twice the size or number will be needed. In addition it may sometimes be necessary to increase the size of air intakes to accommodate the lower suction developed by the fans at the lower speeds. In many instances, however, this will not be necessary as the pressure capability of fans often exceeds amply that required to draw air through the intake.

This means that a fourfold improvement in running cost could be achieved at the expense of roughly twice the capital cost.

\section{SOLAR ELECTRICITY}

The possibility of improving energy efficiency through low-speed operation of fans becomes especially interesting if renewable energy sources are to be used, as electricity from such sources tends to be more expensive than electricity from conventional sources. Since greenhouse cooling fans are mostly used in hot and sunny climates, it is natural to ask whether the fan can be driven from a solar photovoltaic generator (either with back up from the grid, or in stand-alone mode as considered here).

To see what will happen when we couple a photovoltaic generator to the fan motor, we can introduce some equations to represent these components. For the sake of generality, only the simplest equations will be used. We will assume that the motor is a DC type and that the resistance in the motor windings and in the materials of the photovoltaic generator is negligible. For the motor and reduction gear then (Kenjo, 1991):

$$
T=R k_{T} I_{m} \quad[5] \quad \text { and } \quad \omega=v_{m} k_{v} / R
$$


where $T$ is the torque at the fan shaft, $R$ is the reduction ratio, $k_{T}$ the torque constant for the motor, $k_{v}$ the speed constant, $I_{m}$ is the current in the motor and $v_{m}$ the voltage across it. These equations can be combined with Eqs. 2 and 3 to show that the current through the motor will increase with the square of the voltage applied to it, as in Eq. 7 below.

$$
I_{m}=\left(k_{1} k_{v}^{2} / R^{3} k_{T}\right) v_{m}^{2}
$$

The current-voltage curve of the motor can be compared to that for the photovoltaic generator. We represent the photovoltaic generator as a current source in parallel with an ideal diode obeying the Schockley equation (Araújo, 1994). This leads to:

$$
\frac{I_{g}}{I_{s c}}=1-\exp \left[\alpha\left(\frac{v_{g}}{v_{o c}}-1\right)\right]
$$

Here $I_{g}$ is the current in the photovoltaic generator, $I_{s c}$ is the short-circuit current, $v_{g}$ is the voltage across the photovoltaic generator and $v_{o c}$ is the open-circuit voltage. The ratio $\alpha$ is the open-circuit voltage of a single cell in the generator divided by the thermal voltage. For a silicon type cell, as used in the majority of photovoltaic generators, these last two voltages have values of about $0.6 \mathrm{~V}$ and $0.025 \mathrm{~V}$ respectively, giving $\alpha=24$. This is for operation under conditions of bright sunlight, corresponding to an irradiance of 1000 $\mathrm{Wm}^{-2}$. For lower irradiances the $I-v$ curve for the cell is displaced along the $I$ axis. Fig. 2(a) shows a series of curves for a photovoltaic generator operating at different solar irradiations, with the curve for the motor based on Eq. 7 superimposed. The characteristics of the motor and gearing have been chosen so that the two curves cross at a point corresponding to the maximum power output of the generator. In this case the optimisation has been carried out for sunlight of $800 \mathrm{Wm}^{-2}$. At smaller or larger irradiations the matching of the fan to the motor will not be optimised unless a device called a maximum power point (MPP) tracker is used to transform the current and voltage. On the basis of the equations presented above, we have calculated airflow as a function of the irradiation and compared to it that achieved with ideal MPP tracking. The result, shown in Fig. 2(b), shows that in the range of $400 \mathrm{Wm}^{-2}$ to $1000 \mathrm{Wm}^{-2}$, the loss of airflow due to the absence of MPP tracking is less than $10 \%$. For this reason the additional expense and complexity of using the MPP tracking device is hardly justified in this application.

\section{EXPERIMENTAL VERIFICATION}

The above mathematical modelling indicates that more efficient air movement can be obtained at lowered fan speeds and that a fan is well suited to being directly driven from a photovoltaic generator. Since however a number of simplifying assumptions have been made in the modelling, it is desirable to check these findings against real measurements. Unfortunately the fan equipment routinely available from manufacturers does not readily permit low speed operation because it does not come equipped with the appropriate motors and gearing. This practical drawback has been overcome in two ways, as described below.

(i) A variable speed AC drive (Telemecanique $2.2 \mathrm{KW}$, Rueil Malmaison) was connected to two Munters (Stockholm) EM50 1HP fans. Note that this type of drive 
rectifies the incoming mains supply to $\mathrm{DC}$, then reconverts it to $\mathrm{AC}$ at variable frequency for supply to an induction motor. The fans were installed in a fan-and-pad greenhouse near Muscat in the Sultanate of Oman. The power consumption was measured from the inverter display and the air flow through the system measured using a hot-bulb anemometer (Testo 400, Testo AG, Lenzkirch). Rotation speed was measured using an optical tachometer. For comparison against Eqs. 2-4, Figs. 3(a), (b) and (c) show respectively air flow, power consumption and specific energy versus rotation speed.

This fan cooling system was adapted so that the fan speed increased with the level of sunlight. This was achieved by means of a solar sensor connected via a linear amplifier and filter to the speed control input of the AC drive. Fig. 4 compares the power consumption of the variable-speed sunlight-controlled fan, with that of fan running at constant speed from sunrise to sunset. With the system adjusted so that the fan reached full speed at a solar irradiance on the horizontal of $750 \mathrm{Wm}^{-2}$, the electrical power saving from the variable speed arrangement was $40 \%$ for a typical September day.

(ii) A brushless DC motor (Delta Precision Motors 57BL116) was used in place of the standard mains induction motor on a Vostermans (Venlo) Multifan 130, 3-bladed box fan. It was coupled to the fan hub using a lightweight drive belt and pulley arrangement giving a reduction ratio of $6.4: 1$. In this case air flow versus rotation speed was available from the manufacturer, and is shown in Fig. 3(a) for the fan operating at minimal suction (less than $10 \mathrm{~Pa}$ ). Fig. 3(b) shows the power consumption as measured in the laboratory with the unit connected to a bench-top DC power supply.

Fig. 3(a) confirms that the fan law is obeyed in that air flow varies linearly with fan speed. Figs. 3(b) and (c) show that the saving in electrical power at lower speeds is very significant. Compared to operation at the full rated speed, specific energy was reduced by $71 \%$ and $74 \%$ respectively for the EM50 and Multifan 130, close to the value of $75 \%$ predicted by Eq. 4 .

\section{ECONOMIC AND ENVIRONMENTAL EVALUATION}

To assess whether the energy savings from these systems are worthwhile, an economic analysis is needed. The method of analysis chosen here is Net Present Value (NPV), which is a standard way of comparing capital and running costs. However, NPV does not take into account the environmental impacts of the energy usage (ie. external costs). To capture this cost we will incorporate a carbon tax into the calculations. The three systems analysed are: the baseline case, corresponding to a single $750 \mathrm{~W}$ fan running under dawn to dusk conditions; the sunlight-controlled fan [see (i) above] in which the fan is driven by an AC drive connected to a sun sensor; and the solar-powered fan, in which the fan is driven by a DC motor taking electricity from a photovoltaic generator. In this last case, two fans are needed as they are always driven at reduced speeds to minimise the electrical demand on the generator, therefore the capital cost is increased considerably. A 10-year calculation period was used, with discount rates of 0,5 and $10 \%$ has been assumed, with carbon taxes of zero and $€ 50$ per tonne of $\mathrm{CO}_{2}$. Electricity was costed at $€ 0.1 / \mathrm{kWh}$. Some carbon is emitted in the fabrication of the systems, and this was estimated through a simple Life-cycle Analysis using Simapro 7.1 ETH-ESU and IDEMAT databases. Fig. 6 summarises the findings from this analysis. It shows that the sunlight-controlled arrangement is the most economic. High discount rates erode its advantage against the baseline case, while carbon taxation would accentuate its advantage due to the increased energy costs. The solar-powered fan remains expensive under most assumptions. 


\section{DISCUSSION AND RECOMMENDATIONS}

We have shown both theoretically and practically that there is much scope for energy saving in fan-ventilated greenhouses through low speed operation. For example, through running two models of greenhouse fan at half speed we obtained values of specific energy consumption in the range $20-34 \mathrm{Jm}^{-3}$ (ie. $5-10 \mathrm{~W} / 1000 \mathrm{~m}^{3} /$ hour) corresponding to a 71 to $74 \%$ reduction against operation at full rated speed. An inexpensive way of realising these savings is to connect a variable speed drive to the fans, such that full speed is only achieved under bright sunlight and energy is saved when the sun is weaker, giving an average energy saving of about $40 \%$. Powering a greenhouse fan from a photovoltaic generator is an interesting option. The capital cost is high however there are advantage in terms of independence and reliability especially in places such as developing countries where the grid supply may be intermittent. Currently the availability of energy saving fans using low-speed, sunlight-controlled variable speed or solar-power (e.g. using brushless DC motors) is rather poor. Therefore it is recommended that fan manufacturers expand their product range to include such solutions, for example by providing lower gearing ratios on fan drives and integrated electronic speed controls.

\section{ACKNOWLEDGEMENTS}

The authors thank Vostermans BV for providing data used in Fig. 3(a). This work was partly funded by an EPSRC grant reference EP/E044360/1.

\section{Literature cited}

Araújo, G. 1994. Chapter 2: The Solar Cell. In Solar Electricity - Engineering of Photovoltaic Systems, ed. E. Lorenzo, Progensa. Sevilla.

Bleier, F. 1997. Fan Handbook. Chapter 5. McGraw Hill.

Campiglia, E., Colla, G., Mancinelli, R., Rouphael, Y. and Marucci, A. 2007. Energy Balance of Intensive Vegetable Cropping Systems in Central Italy. Acta Hort. 747: 185-191.

Hanan, J. 1998. Greenhouses: Advanced Technology for Protected Horticulture. Chapter 4: Temperature. CRC.

Kenjo, T. 1991. Electric motors and their controls. Oxford University Press. Munters. 2005. Celdek 7090-15. Commercial literature.

Rogers, G. F. C. and Mayhew, Y. R. 1967. Engineering Thermodynamics Work and Heat Transfer. Longman.

Sethi, V. P. and Sharma, S. K. 2008. Survey and evaluation of heating technologies for worldwide agricultural greenhouse applications. Solar Energy, In Press, Corrected Proof.

Sethi, V. P. and Sharma, S. K. 2007. Survey of cooling technologies for worldwide agricultural greenhouse applications. Solar Energy 81: 1447-1459. 
ISHS International Workshop on Greenhouse Environmental Control and Crop Production in Semi-Arid Regions (Tucson, October 2008) Acta Horticulturae, 797, 95-101 (2008)

\section{Figures}

Fig.1: Schematic of the greenhouse cooling arrangement discussed, in which the intake may be an evaporative pad, louvers or other kind of vent.

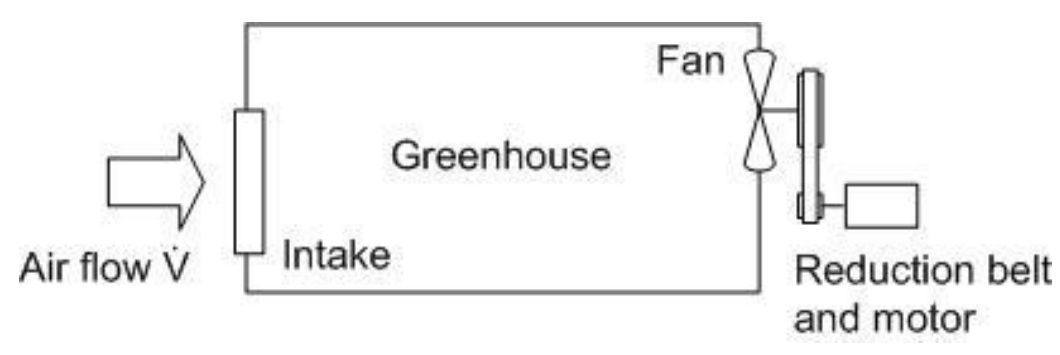

(a)

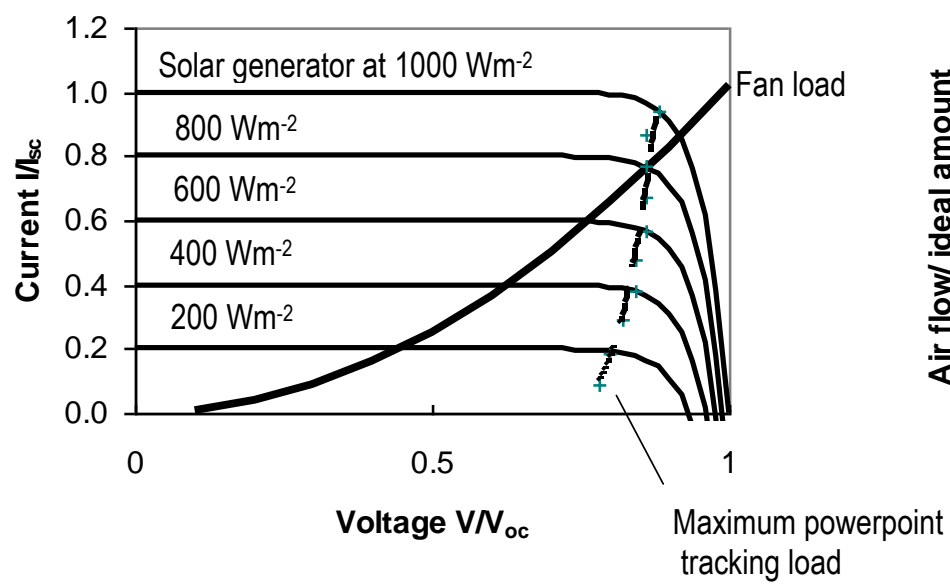

(b)

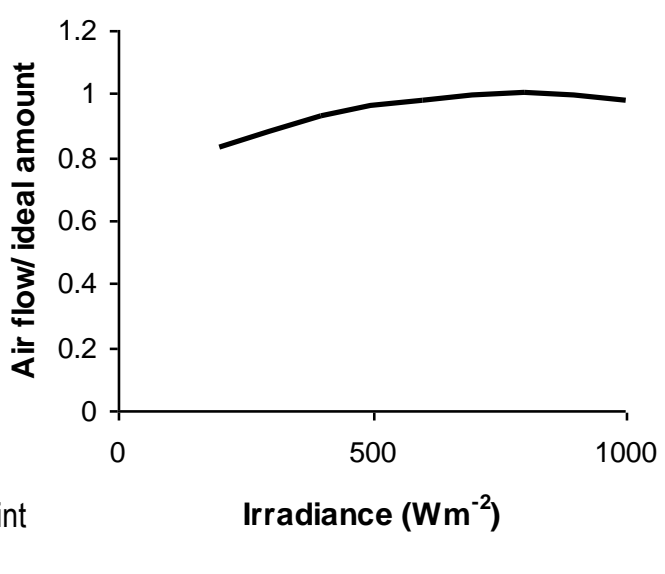

Fig. 2: Based on the simplified model Eqs. 2-8: (a) voltage-current characteristics of the photovoltaic generator and fan load; (b) air flow achieved with the directly coupled generator-motor compared to the ideal amount using maximum power point tracking.

(a)

Fig.3: Comparisons of real fan behaviour (point data) against the fan law (lines) for two fans: a Munters EM50 (-) with a variable speed AC drive and a Vostermans Multifan 130 3-bladed fan ( $\Delta$ ) with a brushless DC motor. (a) Air flow $v s$. fan shaft speed (b) Electrical input power $v s$. fan shaft speed (c) Specific energy $v s$. fan shaft speed.

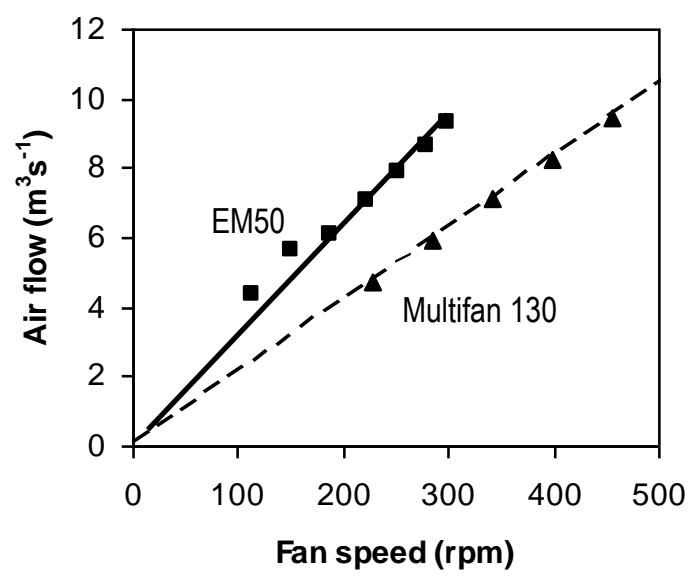

(c)

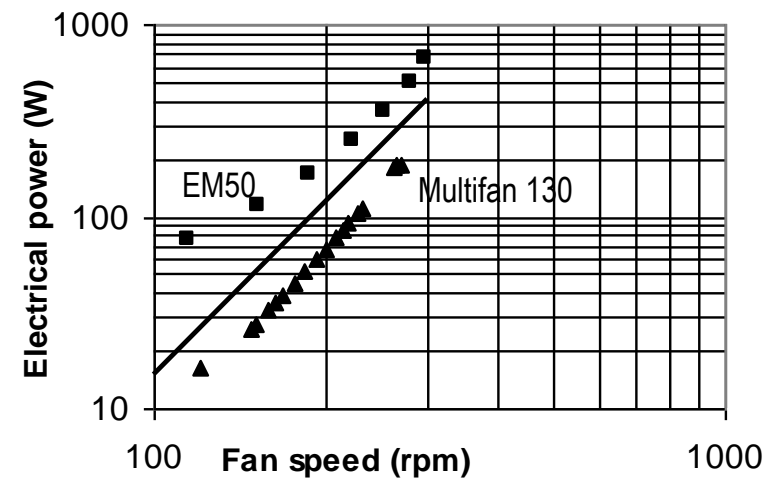

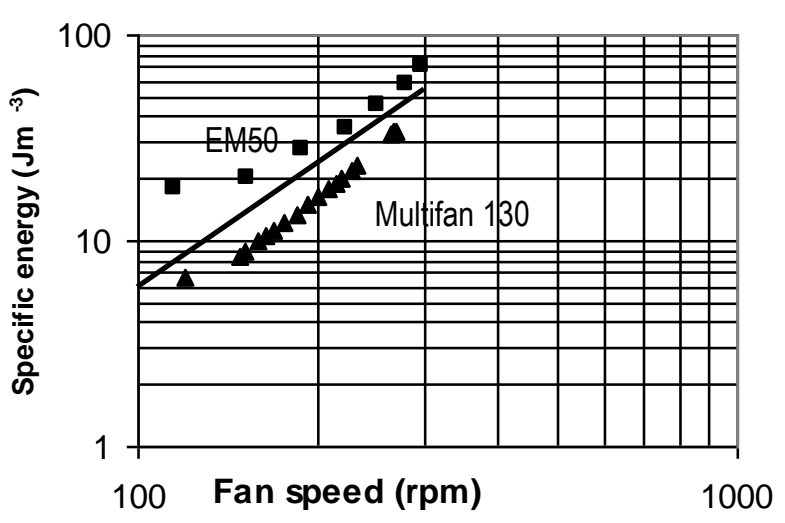


Fig.4: For a Munters EM50 fan installed in a fan-and-pad greenhouse, comparison between sunlightcontrolled variable-speed operation (solid line) and dawn-to-dusk constantspeed operation (broken line). The variable speed operation gives an energy saving of $40 \%$.

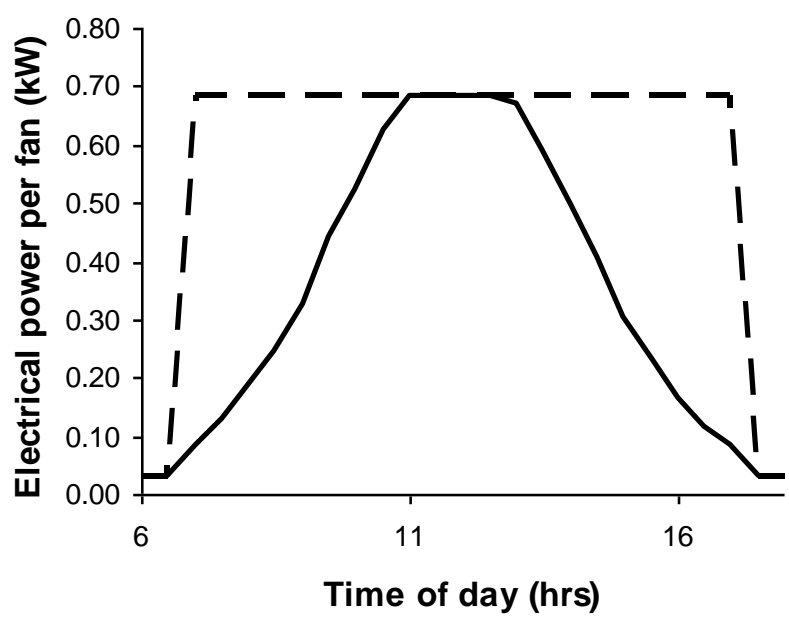

(b) Tax of $€ 50 /$ tonne of $\mathrm{CO} 2$

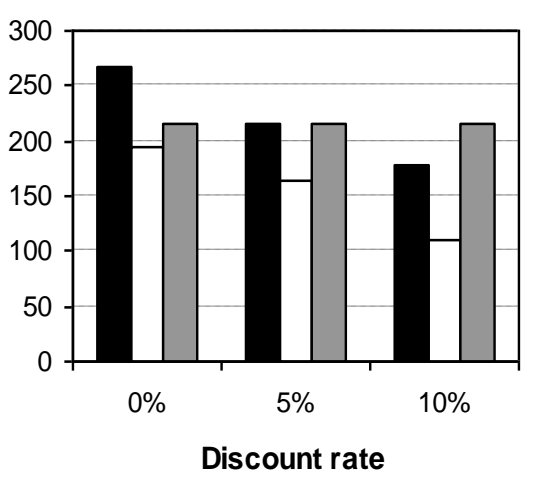

Fig.6: Cost comparisons in euros using Net Present Value of three different arrangements of $1.27 \mathrm{~m}$ diameter fan delivering maximum airflow of $10 \mathrm{~m}^{3} \mathrm{~s}^{-1}$. The baseline case corresponds to a conventional fan operating from dawn till dusk. The sunlight-controlled fan varies in response to sunlight, reaching full speed only around noon and saving power at other times. The solar-powered arrangement will require two fans in the place of the single fans of the preceding cases, running at low speed and using brushless DC motors and photovoltaic panels. 